Journal of Communications and Information Networks, Vol.2, No.4, Dec. 2017

DOI: 10.1007/s41650-017-0036-4

(C) Posts \& Telecom Press and Springer Singapore 2017

\title{
Coverage and rate analysis of user beam selection in mm-wave heterogeneous networks
}

\author{
Tian $\mathrm{Li}^{1}$, Lin $\mathrm{Bai}^{1 *}$, Jinho Choi ${ }^{2}$ \\ 1. School of Electronic and Information Engineering, Beihang University, Beijing 100191, China \\ 2. School of EECS, Gwangju Institute of Science and Technology, Gwangju 61005, Korea \\ * Corresponding author, Email: 1.bai@buaa.edu.cn
}

\begin{abstract}
Millimeter wave (mm-wave) communication is widely considered to be a promising technique for $5 \mathrm{G}$ (Fifth Generation) cellular systems. Owing to the high path loss of mm-wave channels, 5G networks could employ a heterogeneous structure that consists of an MBS (Macro Base Station) and numerous SBSs (Small Base Stations). In this paper, we analyze the coverage and rate performance of an mm-wave heterogeneous network. Using user beam selection, we derive analytical expressions for the coverage probabilities of the SBSs and MBS. Furthermore, the average achievable rate for a typical user is also investigated. It is shown that the analytical results closely follow those of the simulations with marginal differences.
\end{abstract}

Keywords: fifth generation, heterogeneous network, mm-wave, beam selection, coverage probability

Citation: T. Li, L. Bai, J. H. Choi. Coverage and rate analysis of user beam selection in mm-wave heterogeneous networks [J]. Journal of communications and information networks, 2017, 2(4): 120-130.

\section{Introduction}

For developing $5 \mathrm{G}$ cellular systems that can meet the increasingly growing demand of high-speed wireless data services, various techniques have been studied. Among them, mm-wave communication is widely considered a promising method as it can provide a wide available bandwidth ${ }^{[1-4]}$. Compared with other lower-frequency bands (e.g., microwave band), mmwave channels may experience much higher path $\operatorname{loss}^{[5,6]}$. In general, in order to extend the communication range, beamforming can be employed with large-scale arrays at the BS (Base Station) and users $^{[1,7]}$. However, owing to the CSI (Channel State Information) feedback constraint, it is difficult to generate optimized beams at the BS. Thus, as an alternative, beam selection was proposed for perform- ing beamforming, which only requires limited CSI feedback $^{[8,9]}$. In the beam selection scheme, such as the random beamforming approach, both BSs and users generate pre-determined beams. For each BS beam, the users give feedback on the value of the highest SINR (Signal-to-Interference-plus-Noise Ratio) of the receiving beams. The BS then allocates the beam to the user with the best channel condition.

Another specific drawback of mm-wave is the severe blockage effect ${ }^{[10]}$. As signals of higher frequencies are more likely to be blocked by solid materials, the outdoor mm-wave-based BSs may fail to reach indoor users. In this case, it is generally expected that a large number of SBSs should be built in order to create an mm-wave HetNet (Heterogeneous Network) that consists of an MBS and numerous SBSs within a cell[11]. However, as urban areas are

Manuscript received May 17, 2017; accepted Oct. 02, 2017

This work is supported by the National Natural Science Foundation of China (Nos. 61231011, 61231013, and 61571025). 
crowded with high-demand buildings, the SBSs are located in a random manner, which makes the structure of small cellular networks less regular. Fortunately, mathematical tools such as stochastic geometry can offer a solution for the analysis of the performance of such randomly deployed networks ${ }^{[12-15]}$.

In this paper, we focus on the performance analysis of an mm-wave HetNet with user beam selection ${ }^{1)}$ as in Ref. [16]. Based on the results obtained in Ref. [15], we discuss the coverage probabilities of the MBS and SBSs. Furthermore, with the SINR-based user association scheme, the achievable throughput for a user is also considered.

It should be noted that although the discussion is based on Ref. [15], the differences between Ref. [15] and this study can be summarized as follows: i) The system setup in this study is a HetNet that could be a promising structure for $5 \mathrm{G}$, and the analysis thus becomes more challenging. ii) To reduce the user feedback, we apply a beam selection strategy while Ref. [15] considers a single user beam scenario.

As the main contribution of this study, we derive an analytical expression for the coverage probability and average achievable rate of mm-wave HetNets with a beam selection strategy by applying a more general path loss model. Furthermore, the effect of the number of user beams on the coverage probability is theoretically analyzed, which provides an intuitive method for optimizing the system performance. From the expression and simulation results, we find that a better coverage performance can be expected with the beam selection approach by increasing the density of SBSs and the number of user beams, which is different from the results obtained in Ref. [15].

The remainder of the paper is organized as follows. Section 2 presents the system model for an mm-wave HetNet and discusses the SINR at the users. The coverage probabilities obtained with user beam selection are analyzed in section 3. Then, with user association, the achievable rate for a user is derived in section 4. The simulation results are presented and discussed in section 5, and the conclusions of this study are presented in section 6 .

Notation: $\mathcal{C N}(a, v)$ denotes the distribution of a CSCG (Circularly Symmetric Complex Gaussian) random variable with mean $a$ and variance $v$. The statistical expectation and the probability are represented by $\mathbb{E}[\cdot]$ and $\mathbb{P}(\cdot)$, respectively.

\section{System model}

In this section, we describe the system model of a typical mm-wave HetNet with beam selection. Moreover, the SINRs at the users with the use of an MBS and SBSs are also discussed.

\subsection{A typical mm-wave HetNet with SBSs}

The mm-wave HetNet considered in this study is illustrated in Fig. 1, where there exists an MBS and numerous SBSs that are connected by optical cables. We assume that the locations of the SBSs follow a PPP (Poisson Point Process), $\Lambda=\left\{Y_{j}\right\}$, with density $\eta$, and where $Y_{j}$ denotes the $j$ th SBS. Moreover, users that are uniformly located in the macro cell would be served by an SBS or the MBS according to the user association scheme discussed in section 4 . Generally, all the SBSs share the same mm-wave band, which is adjacent to that of the MBS. That is, signals received at a typical user would interfere with other SBSs or the MBS ${ }^{[17]}$.

To compensate for the propagation loss, the BSs and users are equipped with antenna arrays. In particular, considering the implementation constraint and training cost, we assume that the MBS generates near-orthogonal pre-determined beams simultaneously in order to support multiple users. Similarly, an analog beamformer with pre-determined beams is employed at the users. For tractable analysis, the sector beam pattern is considered for approximating

\footnotetext{
1) In this paper, we assume that one of the MBS beams or SBSs has been allocated to a typical user. Thus, the beam selection is simplified to obtain a user beam selection wherein users select the beam with the highest SINR for communicating with the MBS or SBSs.
} 
the beams in this study ${ }^{[15]}$, wherein the main lobe and side lobe gains are denoted as follows:

$G_{S}$ : the main lobe gain of the beams at the SBSs; $g_{S}$ : the side lobe gain of the beams at the SBSs; $G_{M}$ : the main lobe gain of the beams at the MBS; $g_{M}$ : the side lobe gain of the beams at the MBS; $G_{U}$ : the main lobe gain of the beams at the users; $g_{U}$ : the side lobe gain of the beams at the users.

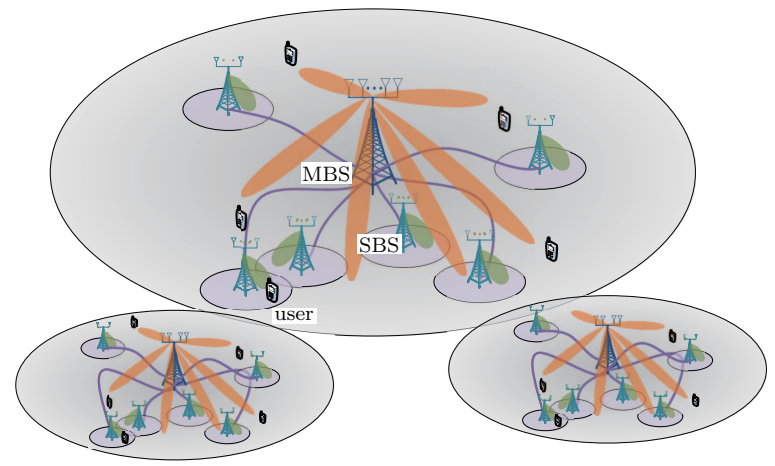

Figure 1 Typical mm-wave HetNet with beam selection

Owing to the blockage effect, the link between a BS beam and a user beam can be modeled as an LOS (Line-of-Sight) or NLOS (non-LOS) link. Hence, with a link length $r$, the path loss can be calculated as

$$
L(r)= \begin{cases}\beta_{L}\left(1+r^{\alpha_{L}}\right)^{-1}, & \text { w.p. } p_{L}(r), \\ \beta_{N}\left(1+r^{\alpha_{N}}\right)^{-1}, & \text { w.p. } 1-p_{L}(r),\end{cases}
$$

where $\beta_{L}$ and $\beta_{N}$ denote the signal attenuations with $r=0$ for LOS and NLOS links, respectively. The path loss exponents of LOS and NLOS links are represented by $\alpha_{L}$ and $\alpha_{N}$, respectively. It should be noted that $p_{L}(r)=\mathrm{e}^{-\mu r}$ denotes the probability of an LOS link, which has been discussed in Ref. [14].

Remark 1: The path loss, $\beta r^{-\alpha}$ (we use $\alpha$ instead of $\alpha_{L}$ or $\alpha_{N}$ for notational simplicity), employed in Ref. [14] might not be appropriate when $0 \leqslant r<1$. Thus, without the loss of generality, we use $\beta\left(1+r^{\alpha}\right)^{-1}$ instead of $\beta r^{-\alpha}$ in this study ${ }^{[18]}$.

\subsection{SINRs of different associated BSs}

Owing to implementation difficulties and computational complexity, we consider an analog beamformer with fixed beams at the users. In this case, the beam associated with the highest SINR must be selected for realizing an optimal performance.

Let us suppose that each user has a codebook with $N_{U}$ pre-determined beams. If a user is served by an SBS, the SINR of the $i$ th user beam is given by

$$
S I N R_{i, S}=\frac{\left|h_{0 ; i}\right|^{2} G_{U} G_{S} L\left(r_{0 ; i}\right) P_{S}}{\sigma^{2}+I_{i, S}+I_{i, M} / \rho},
$$

where

$$
I_{i, S}=\sum_{j>0, Y_{j} \in \Lambda}\left|h_{j ; i}\right|^{2} D_{j ; i} L\left(r_{j ; i}\right) P_{S}
$$

and

$$
I_{i, M}=\sum_{l=1}^{K}\left|h_{l ; i}\right|^{2} T_{l ; i} L\left(x_{M}\right) P_{M} / K .
$$

Here, $K$ denotes the number of fixed beams at the MBS, and $x_{M}$ represents the distance of the user from the MBS. $h_{j ; i} \sim \mathcal{C N}(0,1)$ and $h_{b ; i} \sim \mathcal{C N}(0,1)$ denote the small-scale fading coefficients related to the $j$ th SBS and $b$ th MBS beam at the $i$ th user beam, respectively. Moreover, the transmission powers at the SBSs and MBS are denoted by $P_{S}$ and $P_{M}$, respectively, while $\sigma^{2}$ denotes the background noise power. The adjacent channel selectivity is represented by $\rho$. Let $D_{j ; i}$ denote the link gain from the $j$ th SBS to the $i$ th user beam. Then, $D_{j ; i}=D$, $i \in\left\{1, \cdots, N_{U}\right\}$ can be modeled as a random variable with the distribution as $D=\nu_{k}$ with probability $a_{k}, k \in\{1,2,3,4\}$, as shown in Tab. 1 .

Table 1 The distribution of $D$

\begin{tabular}{ccccc}
\hline$\nu_{k}$ & $G_{U} G_{S}$ & $G_{U} g_{S}$ & $g_{U} G_{S}$ & $g_{U} g_{S}$ \\
\hline$a_{k}$ & $\frac{\theta_{U}}{2 \pi} \frac{\theta_{S}}{2 \pi}$ & $\frac{\theta_{U}}{2 \pi}\left(1-\frac{\theta_{S}}{2 \pi}\right)$ & $\left(1-\frac{\theta_{U}}{2 \pi}\right) \frac{\theta_{S}}{2 \pi}$ & $\left(1-\frac{\theta_{U}}{2 \pi}\right)\left(1-\frac{\theta_{S}}{2 \pi}\right)$ \\
\hline
\end{tabular}

It should be noted that in Tab. $1, \theta_{S}$ and $\theta_{U}$ denote the half power beamwidths of the SBSs and users, respectively. Similarly, by defining the beamwidth of each fixed beam at the MBS as $\theta_{M}$, the distribution for the link gain $T_{l ; i}=T, l \in\{1, \cdots, K\}$, can be derived, as shown in Tab. 2. 
Table 2 The distribution of $T$

\begin{tabular}{ccccc}
\hline$\xi_{k}$ & $G_{U} G_{M}$ & $G_{U} g_{M}$ & $g_{U} G_{M}$ & $g_{U} g_{M}$ \\
\hline$b_{k} \frac{\theta_{U}}{2 \pi} \frac{\theta_{M}}{2 \pi}$ & $\frac{\theta_{U}}{2 \pi}\left(1-\frac{\theta_{M}}{2 \pi}\right)$ & $\left(1-\frac{\theta_{U}}{2 \pi}\right) \frac{\theta_{M}}{2 \pi}$ & $\left(1-\frac{\theta_{U}}{2 \pi}\right)\left(1-\frac{\theta_{M}}{2 \pi}\right)$
\end{tabular}

In contrast, if the $i$ th user is connected to the MBS, the SINR with MBS beam $m$ and user beam $i$ becomes

$$
S I N R_{i, M}=\frac{\left|h_{m ; i}\right|^{2} G_{U} G_{M} L\left(x_{M}\right) P_{M} / K}{\sigma^{2}+I_{i, S}^{\prime} / \rho+I_{i, M}^{\prime}}
$$

where

$$
I_{i, S}^{\prime}=\sum_{j, Y_{j} \in \Lambda}\left|h_{j ; i}\right|^{2} D_{j ; i} L\left(r_{j ; i}\right) P_{S}
$$

and

$$
I_{i, M}^{\prime}=\sum_{l \neq m}\left|h_{l ; i}\right|^{2} T_{l ; i} L\left(x_{M}\right) P_{M} / K .
$$

Based on Eqs. (2) and (5), we analyze the coverage probabilities of the HetNet in section 3.

Remark 2: In the case of the IMCI (Inter-MacroCell Interference), owing to the large distances between inter-MBSs and users, we only consider the IMCI of the SBSs.

\section{Coverage probability analysis with user beam selection}

For the coverage analysis with user beam selection, we provide the following definition.

Definition 1 Given an SINR threshold $\Gamma$, the coverage probability is defined by

$$
P_{\text {cov }}(\Gamma)=\mathbb{P}\left(\max _{1 \leqslant i \leqslant N_{U}} S I N R_{i} \geqslant \Gamma\right) .
$$

According to Eq. (8), when a user is served by an SBS, the coverage probability can be expressed as

$$
\begin{aligned}
& P_{\mathrm{cov}, S}(\Gamma) \\
= & \mathbb{P}\left(\max _{1 \leqslant i \leqslant N_{U}} S I N R_{i, S} \geqslant \Gamma\right) \\
= & 1-\mathbb{P}\left(S I N R_{1, S}<\Gamma, \ldots, S I N R_{N_{U}, S}<\Gamma\right) .
\end{aligned}
$$

Assumption 1 As the analog beamformer is employed at the users, a fixed beam is generated in a single time slot. Thus, for tractable analysis, we assume that the SBSs are independent for the $N_{U}$ user beams.

Given that the user is associated with an SBS, the probability that such an SBS has an LOS link can be calculated as ${ }^{[15]}$

$$
\Phi_{L}=\Psi_{L} \int_{0}^{\infty} \mathrm{e}^{-2 \pi \eta \int_{0}^{\varepsilon_{L}(r)}\left(1-p_{L}(t)\right) t \mathrm{~d} t} \phi_{L}(r) \mathrm{d} r,(10)
$$

where

$$
\varepsilon_{L}(r)=\left(\left(\beta_{N} / \beta_{L}\right)\left(1+r^{\alpha_{L}}\right)-1\right)^{1 / \alpha_{N}}
$$

and $\Psi_{L}=1-\mathrm{e}^{-2 \pi \eta \int_{0}^{\infty} x p_{L}(x) \mathrm{d} x}$. Furthermore, $\phi_{L}(r)=2 \pi \eta r p_{L}(r) \mathrm{e}^{-2 \pi \eta \int_{0}^{r} x p_{L}(x) \mathrm{d} x} / \Psi_{L}$ denotes the PDF (Probability Density Function) for the distance between a user and the nearest LOS SBS. In this case, the probability that the user is served by an NLOS SBS is $\Phi_{N}=1-\Phi_{L}$.

Let us suppose that the radius of the macro cell is denoted by $R$. We then obtain the following results.

Lemma 1 The coverage probability $P_{\mathrm{cov}, S}(\Gamma)$ can be calculated as

$$
P_{\mathrm{cov}, S}(\Gamma)=1-\int_{0}^{R}\left(1-\left(\Phi_{L} W\left(\Gamma, x_{M}\right)+\Phi_{N} V\left(\Gamma, x_{M}\right)\right)\right)^{N_{U}} \frac{2 x_{M}}{R^{2}} \mathrm{~d} x_{M},
$$

where

$$
\begin{gathered}
W\left(\Gamma, x_{M}\right)=\int_{0}^{\infty} \mathrm{e}^{-\frac{\Gamma \sigma^{2}}{G_{U} G_{S} \beta_{L}\left(1+r^{\alpha} L\right)-1} P_{S}} J_{S}(\Gamma, r) J_{M}\left(\Gamma, r, x_{M}\right) f_{L}(r) \mathrm{d} r, \\
V\left(\Gamma, x_{M}\right)=\int_{0}^{\infty} \mathrm{e}^{-\frac{\Gamma \sigma^{2}}{G_{U} G_{S} \beta_{N}\left(1+r^{\alpha}\right)^{-1} P_{S}}} Q_{S}(\Gamma, r) Q_{M}\left(\Gamma, r, x_{M}\right) f_{N}(r) \mathrm{d} r .
\end{gathered}
$$


In Eqs. (13) and (14), we have

$$
\begin{aligned}
& J_{S}(\Gamma, r)=\exp \left(-2 \pi \eta \int_{r}^{\infty}\left(1-\sum_{k=1}^{4} a_{k} \frac{1}{1+\frac{\Gamma \nu_{k}\left(1+t^{\alpha_{L}}\right)^{-1}}{G_{U} G_{S}\left(1+r^{\alpha_{L}}\right)^{-1}}}\right) p_{L}(t) t \mathrm{~d} t\right) \\
& \exp \left(-2 \pi \eta \int_{\varepsilon_{L}(r)}^{\infty}\left(1-\sum_{k=1}^{4} a_{k} \frac{1}{1+\frac{\Gamma \nu_{k} \beta_{N}\left(1+t^{\alpha_{N}}\right)^{-1}}{G_{U} G_{S} \beta_{L}\left(1+r^{\alpha_{L}}\right)^{-1}}}\right)\left(1-p_{L}(t)\right) t \mathrm{~d} t\right), \\
& J_{M}\left(\Gamma, r, x_{M}\right)=\left(\sum_{k=1}^{4} b_{k} \frac{1}{1+\frac{\Gamma \xi_{k}\left(1+x_{M}^{\alpha_{L}}\right)^{-1} P_{M} /(\rho K)}{G_{U} G_{S}\left(1+r^{\alpha_{L}}\right)^{-1} P_{S}}} p_{L}\left(x_{M}\right)\right. \\
& \left.+\sum_{k=1}^{4} b_{k} \frac{1}{1+\frac{\Gamma \xi_{k} \beta_{N}\left(1+x_{M}^{\alpha_{N}}\right)^{-1} P_{M} /(\rho K)}{G_{U} G_{S} \beta_{L}\left(1+r^{\alpha_{L}}\right)^{-1} P_{S}}}\left(1-p_{L}\left(x_{M}\right)\right)\right)^{K}, \\
& Q_{S}(\Gamma, r)=\exp \left(-2 \pi \eta \int_{\varepsilon_{N}(r)}^{\infty}\left(1-\sum_{k=1}^{4} a_{k} \frac{1}{1+\frac{\Gamma \nu_{k} \beta_{L}\left(1+t^{\alpha_{L}}\right)^{-1}}{G_{U} G_{S} \beta_{N}\left(1+r^{\alpha_{N}}\right)^{-1}}}\right) p_{L}(t) t \mathrm{~d} t\right) \\
& \times \exp \left(-2 \pi \eta \int_{r}^{\infty}\left(1-\sum_{k=1}^{4} a_{k} \frac{1}{1+\frac{\Gamma \nu_{k}\left(1+t^{\alpha_{N}}\right)^{-1}}{G_{U} G_{S}\left(1+r^{\alpha_{N}}\right)^{-1}}}\right)\left(1-p_{L}(t)\right) t \mathrm{~d} t\right),
\end{aligned}
$$

and

$$
\begin{aligned}
Q_{M}\left(\Gamma, r, x_{M}\right)= & \left(\sum_{k=1}^{4} b_{k} \frac{1}{1+\frac{\Gamma \xi_{k} \beta_{L}\left(1+x_{M}^{\alpha_{L}}\right)^{-1} P_{M} /(\rho K)}{G_{U} G_{S} \beta_{N}\left(1+r^{\alpha_{N}}\right)^{-1} P_{S}}} p_{L}\left(x_{M}\right)\right. \\
& \left.+\sum_{k=1}^{4} b_{k} \frac{1}{1+\frac{\Gamma \xi_{k}\left(1+x_{M}^{\alpha_{N}}\right)^{-1} P_{M} /(\rho K)}{G_{U} G_{S}\left(1+r^{\alpha_{N}}\right)^{-1} P_{S}}}\left(1-p_{L}\left(x_{M}\right)\right)\right)^{K},
\end{aligned}
$$

where $\varepsilon_{N}(r)=\left(\left(\beta_{L} / \beta_{N}\right)\left(1+r^{\alpha_{N}}\right)-1\right)^{1 / \alpha_{L}}$. The PDF of the distance to the serving LOS and NLOS SBSs are denoted by $f_{L}(r)$ and $f_{N}(r)$, respectively, and they can be obtained as

$$
\begin{gathered}
f_{L}(r)=\frac{\Psi_{L}}{\Phi_{L}} \phi_{L}(r) \mathrm{e}^{-2 \pi \eta \int_{0}^{\varepsilon_{L}(r)}\left(1-p_{L}(t)\right) t \mathrm{~d} t}, \\
f_{N}(r)=\frac{\Psi_{N}}{\Phi_{N}} \phi_{N}(r) \mathrm{e}^{-2 \pi \eta \int_{0}^{\varepsilon_{N}(r)} p_{L}(t) t \mathrm{~d} t},
\end{gathered}
$$

where

$$
\Psi_{N}=1-\mathrm{e}^{-2 \pi \eta \int_{0}^{\infty} x\left(1-p_{L}(x)\right) \mathrm{d} x} .
$$

Similarly, $\phi_{N}(r)=2 \pi \eta r p_{L}(r) \mathrm{e}^{-2 \pi \eta \int_{0}^{r} x\left(1-p_{L}(x)\right) \mathrm{d} x} / \Psi_{N}$ represents the PDF of the distance between a user and the nearest NLOS SBS. 
Proof Based on Assumption 1, Eq. (9) becomes

$$
\begin{aligned}
P_{\operatorname{cov}, S}(\Gamma) & =1-\int_{0}^{R} \prod_{i=1}^{N_{U}} \mathbb{P}\left(S I N R_{i, S}<\Gamma\right) \frac{2 x_{M}}{R^{2}} \mathrm{~d} x_{M}=1-\int_{0}^{R}\left(1-\mathbb{E}\left[\mathrm{e}^{-\frac{\Gamma\left(\sigma^{2}+I_{i, S}+I_{i, M} / \rho\right)}{G_{U} G_{S} L\left(r_{0 ;}\right) P_{S}}}\right]\right)^{N_{U}} \frac{2 x_{M}}{R^{2}} \mathrm{~d} x_{M} \\
& =1-\int_{0}^{R}\left(1-\left(\Phi_{L} W\left(\Gamma, x_{M}\right)+\Phi_{N} V\left(\Gamma, x_{M}\right)\right)\right)^{N_{U}} \frac{2 x_{M}}{R^{2}} \mathrm{~d} x_{M}
\end{aligned}
$$

where

$$
\begin{aligned}
W\left(\Gamma, x_{M}\right) & =\mathbb{E}\left[\mathrm{e}^{-\frac{\Gamma\left(\sigma^{2}+I_{i, S}+I_{i, M} / \rho\right)}{G_{U} G_{S} \beta_{L}\left(1+r_{0 ; i}^{\alpha}\right)^{L}-1 P_{S}}}\right] \\
& =\int_{0}^{\infty} \mathrm{e}^{-\frac{\Gamma \sigma^{2}}{G_{U} G_{S} \beta_{L}\left(1+r^{\alpha} L\right)-1} P_{S}} \mathbb{E}\left[\mathrm{e}^{-\frac{\Gamma I_{i, S}}{G_{U} G_{S} \beta_{L}\left(1+r^{\alpha} L\right)-1} P_{S}}\right] \mathbb{E}\left[\mathrm{e}^{-\frac{\Gamma I_{i, M} / \rho}{G_{U} G_{S} \beta_{L}\left(1+r^{2} L\right)-1} P_{S}}\right] f_{L}(r) \mathrm{d} r .
\end{aligned}
$$

On dividing the interference into LOS and NLOS parts, we obtain

$$
\mathbb{E}\left[\mathrm{e}^{-\frac{\Gamma I_{i, S}}{G_{U} G_{S} \beta_{L}\left(1+r^{\alpha} L\right)^{-1} P_{S}}}\right]=\mathbb{E}\left[\mathrm{e}^{-\frac{\Gamma \sum_{l}\left|h_{l ;}\right|^{2} D_{l ; i}\left(1+r_{l}^{\alpha} L\right)^{-1}}{G_{U} G_{S}\left(1+r^{\alpha} L\right)^{-1}}}\right] \mathbb{E}\left[\mathrm{e}^{-\frac{\Gamma \sum_{n}\left|h_{n ; i}\right|^{2} D_{n ; i} \beta_{N}\left(1+r_{n}^{\alpha} N\right)^{-1}}{G_{U} G_{S} \beta^{\beta}\left(1+r^{\alpha} L\right)^{-1}}}\right] .
$$

Based on the moment-generating function of an exponential random variable, Eq. (24) can be rewritten as

$$
\mathbb{E}\left[\mathrm{e}^{-\frac{\Gamma I_{i, S}}{G_{U} G_{S} \beta_{L}\left(1+r^{\alpha_{L}}\right)^{-1} P_{S}}}\right]=\mathbb{E}\left[\prod_{l} \frac{1}{1+\frac{\Gamma D_{l ; i}\left(1+r_{l}^{\alpha_{L}}\right)^{-1}}{G_{U} G_{S}\left(1+r^{\alpha_{L}}\right)^{-1}}}\right] \mathbb{E}\left[\prod_{n} \frac{1}{1+\frac{\Gamma D_{n ; i} \beta_{N}\left(1+r_{n}^{\alpha_{N}}\right)^{-1}}{G_{U} G_{S} \beta_{L}\left(1+r^{\alpha_{L}}\right)^{-1}}}\right] .
$$

Based on Tab. 1 and the Laplace functional of a PPP, Eq. (25) can be calculated as

$$
\begin{aligned}
& \mathbb{E}\left[\mathrm{e}^{\left.-\frac{\Gamma I_{i, S}}{G_{U} G_{S} \beta_{L}\left(1+r^{\alpha_{L}}\right)^{-1} P_{S}}\right]}\right. \\
= & \mathbb{E}\left[\prod_{l} \sum_{k=1}^{4} \alpha_{k} \frac{1}{1+\frac{\Gamma \nu_{l ; i, k}\left(1+r_{l}^{\alpha_{L}}\right)^{-1}}{G_{U} G_{S}\left(1+r^{\alpha_{L}}\right)^{-1}}}\right] \mathbb{E}\left[\prod_{n} \sum_{k=1}^{4} \alpha_{k} \frac{1}{1+\frac{\Gamma \nu_{n ; i, k} \beta_{N}\left(1+r_{n}^{\alpha_{N}}\right)^{-1}}{G_{U} G_{S} \beta_{L}\left(1+r^{\alpha_{L}}\right)^{-1}}}\right] \\
= & \exp \left(-2 \pi \eta \int_{r}^{\infty}\left(1-\sum_{k=1}^{4} a_{k} \frac{1}{1+\frac{\Gamma \nu_{i, k}\left(1+t^{\alpha_{L}}\right)^{-1}}{G_{U} G_{S}\left(1+r^{\alpha_{L}}\right)^{-1}}}\right) p_{L}(t) t \mathrm{~d} t\right) \\
& \cdot \exp \left(-2 \pi \eta \int_{\varepsilon_{L}(r)}^{\infty}\left(1-\sum_{k=1}^{4} a_{k} \frac{\Gamma}{1+\frac{\Gamma \nu_{i, k} \beta_{N}\left(1+t^{\alpha_{N}}\right)^{-1}}{G_{U} G_{S} \beta_{L}\left(1+r^{\alpha_{L}}\right)^{-1}}}\right)\left(1-p_{L}(t)\right) t \mathrm{~d} t\right) .
\end{aligned}
$$

Thus, we obtain Eq. (15).

For $\mathbb{E}\left[\mathrm{e}^{-\frac{\Gamma I_{i, M} / \rho}{G_{U} G_{S}{ }^{\beta}\left(1+r^{\alpha} L\right)-1} P_{S}}\right]$, we have

$$
\mathbb{E}\left[\mathrm{e}^{-\frac{\Gamma I_{i, M} / \rho}{G_{U} G_{S} \beta_{L}\left(1+r^{\alpha} L\right)^{-1} P_{S}}}\right]=\left(\mathbb{E}\left[\mathrm{e}^{-\frac{\Gamma\left|h_{l ; i}\right|^{2} T_{l ;} L\left(x_{M}\right) P_{M} /(\rho K)}{G_{U} G_{S} \beta_{L}\left(1+r^{\alpha} L\right)-1} P_{S}}\right]\right)^{K}
$$




$$
\begin{aligned}
& =\left(\mathbb{E}\left[\mathrm{e}^{-\frac{\Gamma\left|h_{l ; i}\right|^{2} T_{l ; i}\left(1+x_{M}^{\alpha}\right)^{-1} P_{M} /(\rho K)}{G_{U} G_{S}\left(1+r^{\alpha} L\right)^{-1} P_{S}}}\right] p_{L}\left(x_{M}\right)\right. \\
& \left.+\mathbb{E}\left[\mathrm{e}^{-\frac{\Gamma\left|h_{l ; i}\right|^{2} T_{l ; i} \beta_{N}\left(1+x_{M}^{\alpha}\right)^{-1} P_{M} /(\rho K)}{G_{U} G_{S} \beta_{L}\left(1+r^{\alpha} L\right)^{-1} P_{S}}}\right]\left(1-p_{L}\left(x_{M}\right)\right)\right)^{K} .
\end{aligned}
$$

Then, based on the aforementioned moment-generating function, we can derive Eq. (16). When the user is associated with an NLOS SBS, $V\left(\Gamma, x_{M}\right)$ can be proved to be Eq. (14) in a manner similar to that discussed above.

Property 1 The coverage probability in Eq. (12) increases with $N_{U}$ as a higher spatial diversity gain can be exploited.

Proof Let $H\left(x_{M}\right)=1-\left(\Phi_{L} W\left(\Gamma, x_{M}\right)+\Phi_{N} V\left(\Gamma, x_{M}\right)\right)$ and $N_{U}$ is set as a variable. Then, Eq. (12) becomes

$$
P_{\text {cov }, S}\left(N_{U}\right)=1-\int_{0}^{R} H\left(x_{M}\right)^{N_{U}} \frac{2 x_{M}}{R^{2}} \mathrm{~d} x_{M} .
$$

Next, let $\Upsilon=P_{\operatorname{cov}, S}\left(N_{U}\right)-P_{\operatorname{cov}, S}\left(N_{U}-1\right)$. We obtain

$$
\begin{aligned}
\Upsilon & =1-\int_{0}^{R} H\left(x_{M}\right)^{N_{U}} \frac{2 x_{M}}{R^{2}} \mathrm{~d} x_{M}-\left(1-\int_{0}^{R} H\left(x_{M}\right)^{N_{U}-1} \frac{2 x_{M}}{R^{2}} \mathrm{~d} x_{M}\right) \\
& =\int_{0}^{R} \frac{2 x_{M}}{R^{2}} H\left(x_{M}\right)^{N_{U}}\left(H\left(x_{M}\right)^{-1}-1\right) \mathrm{d} x_{M} .
\end{aligned}
$$

As $H\left(x_{M}\right)<1$, we have $H\left(x_{M}\right)^{-1}-1>0$. Thus, $\Upsilon>0$, which indicates that the coverage probability increases with $N_{U}$.

Lemma 2 If the user is served by the MBS, the coverage probability is given by

$$
P_{\mathrm{cov}, M}(\Gamma)=1-\int_{0}^{R}\left(1-F_{L}\left(\Gamma, x_{M}\right) p_{L}\left(x_{M}\right)-F_{N}\left(\Gamma, x_{M}\right)\left(1-p_{L}\left(x_{M}\right)\right)\right)^{N_{U}} \frac{2 x_{M}}{R^{2}} \mathrm{~d} x_{M},
$$

where

$$
\begin{gathered}
F_{L}\left(\Gamma, x_{M}\right)=\mathrm{e}^{-\frac{K \Gamma \sigma^{2}}{G_{U} G_{M} \beta_{L}\left(1+x_{M}^{\alpha}\right)^{-1} P_{M}}} U_{S}\left(\Gamma, x_{M}\right) U_{M}\left(\Gamma, x_{M}\right), \\
F_{N}\left(\Gamma, x_{M}\right)=\mathrm{e}^{-\frac{K \Gamma \sigma^{2}}{G_{U} G_{M} \beta_{N}\left(1+x_{M}^{\alpha}\right)^{-1} P_{M}}} Z_{S}\left(\Gamma, x_{M}\right) Z_{M}\left(\Gamma, x_{M}\right) .
\end{gathered}
$$

In Eqs. (31) and (32), $U_{S}\left(\Gamma, x_{M}\right), U_{M}\left(\Gamma, x_{M}\right), Z_{S}\left(\Gamma, x_{M}\right)$, and $Z_{M}\left(\Gamma, x_{M}\right)$ are given as follows:

$$
\left.\begin{array}{rl}
U_{S}\left(\Gamma, x_{M}\right)= & \exp \left(-2 \pi \eta \int_{0}^{\infty}\left(1-\sum_{k=1}^{4} a_{k} \frac{1}{1+\frac{\Gamma \nu_{k}\left(1+r^{\alpha_{L}}\right)^{-1} P_{S} / \rho}{G_{U} G_{M}\left(1+x_{M}^{\alpha_{L}}\right)^{-1} P_{M} / K}}\right) p_{L}(r) r \mathrm{~d} r\right) \\
& \cdot \exp \left(-2 \pi \eta \int_{0}^{\infty}\left(1-\sum_{k=1}^{4} a_{k} \frac{1}{1+\frac{\Gamma \nu_{k} \beta_{N}\left(1+r^{\alpha_{N}}\right)^{-1} P_{S} / \rho}{G_{U} G_{M} \beta_{L}\left(1+x_{M}^{\alpha_{L}}\right)^{-1} P_{M} / K}}\right)\left(1-p_{L}(r)\right) r \mathrm{~d} r\right) \\
U_{M}\left(\Gamma, x_{M}\right)= & \left(\sum_{k=1}^{4} b_{k} \frac{1}{1+\frac{\Gamma \xi_{k}}{G_{U} G_{M}}} p_{L}\left(x_{M}\right)+\sum_{k=1}^{4} \frac{1}{1+\frac{\Gamma \xi_{k} \beta_{N}\left(1+x_{M}^{\alpha_{N}}\right)^{-1}}{G_{U} G_{M} \beta_{L}\left(1+x_{M}^{\alpha_{L}}\right)^{-1}}}\left(1-p_{L}\left(x_{M}\right)\right)\right.
\end{array}\right)
$$




$$
\begin{aligned}
Z_{S}\left(\Gamma, x_{M}\right)= & \exp \left(-2 \pi \eta \int_{0}^{\infty}\left(1-\sum_{k=1}^{4} a_{k} \frac{1}{1+\frac{\Gamma \nu_{k} \beta_{L}\left(1+r^{\alpha_{L}}\right)^{-1} P_{S} / \rho}{G_{U} G_{M} \beta_{N}\left(1+x_{M}^{\alpha_{N}}\right)^{-1} P_{M} / K}}\right) p_{L}(r) r \mathrm{~d} r\right) \\
& \cdot \exp \left(-2 \pi \eta \int_{0}^{\infty}\left(1-\sum_{k=1}^{4} a_{k} \frac{1}{1+\frac{\Gamma \nu_{k}\left(1+r^{\alpha_{N}}\right)^{-1} P_{S} / \rho}{G_{U} G_{M}\left(1+x_{M}^{\alpha_{N}}\right)^{-1} P_{M} / K}}\right)\left(1-p_{L}(r)\right) r \mathrm{~d} r\right),
\end{aligned}
$$

and

$$
Z_{M}\left(\Gamma, x_{M}\right)=\left(\sum_{k=1}^{4} b_{k} \frac{1}{1+\frac{\Gamma \xi_{k} \beta_{L}\left(1+x_{M}^{\alpha_{L}}\right)^{-1}}{G_{U} G_{M} \beta_{N}\left(1+x_{M}^{\alpha_{N}}\right)^{-1}}} p_{L}\left(x_{M}\right)+\sum_{k=1}^{4} b_{k} \frac{1}{1+\frac{\Gamma \xi_{k}}{G_{U} G_{M}}}\left(1-p_{L}\left(x_{M}\right)\right)\right)^{K-1} .
$$

Proof According to Eqs. (5) and (8), $P_{\operatorname{cov}, M}(\Gamma)$ is given by

$$
P_{\text {cov }, M}(\Gamma)=\mathbb{P}\left(\max _{1 \leqslant i \leqslant N_{U}} S I N R_{i, M} \geqslant \Gamma\right)=1-\int_{0}^{R}\left(1-\mathbb{E}\left[\mathrm{e}^{-\frac{\Gamma\left(\sigma^{2}+I_{i, S}^{\prime} / \rho+I_{i, M}^{\prime}\right)}{G_{U} G_{M} L\left(x_{M}\right) P_{M} / K}}\right]\right)^{N_{U}} \frac{2 x_{M}}{R^{2}} \mathrm{~d} x_{M} .
$$

As in Eq. (22), Eq. (37) can be calculated as

$$
P_{\mathrm{cov}, M}(\Gamma)=1-\int_{0}^{R}\left(1-F_{L}\left(\Gamma, x_{M}\right) p_{L}\left(x_{M}\right)-F_{N}\left(\Gamma, x_{M}\right)\left(1-p_{L}\left(x_{M}\right)\right)\right)^{N_{U}} \frac{2 x_{M}}{R^{2}} \mathrm{~d} x_{M},
$$

where

$$
\begin{aligned}
F_{L}\left(\Gamma, x_{M}\right) & =\mathbb{E}\left[\mathrm{e}^{-\frac{\Gamma\left(\sigma^{2}+I_{i, S}^{\prime} / \rho+I_{i, M}^{\prime}\right)}{G_{U} G_{M} \beta_{L}\left(1+x_{M}^{\alpha}\right)-1 P_{M} / K}}\right] \\
& =\mathrm{e}^{-\frac{K \Gamma \sigma^{2}}{G_{U} G_{M} \beta_{L}\left(1+x_{M}^{\alpha}\right)-1 P_{M}}} \mathbb{E}\left[\mathrm{e}^{-\frac{K \Gamma I_{i, S}^{\prime} / \rho}{G_{U} G_{M} \beta_{L}\left(1+x_{M}^{\alpha}\right)-1 P_{M}}}\right] \mathbb{E}\left[\mathrm{e}^{-\frac{K \Gamma I_{i, M}^{\prime}}{G_{U} G_{M} \beta_{L}\left(1+x_{M}\right)^{-1} P_{M}}}\right] .
\end{aligned}
$$

Here, $\mathbb{E}\left[\mathrm{e}^{-\frac{K \Gamma I_{i, S}^{\prime} / \rho}{G_{U} G_{M} \beta_{L}\left(1+x_{M}^{\alpha}\right)^{-1} P_{M}}}\right]$ can be derived as Eq. (33) in a manner similar to that in Proof 1. According

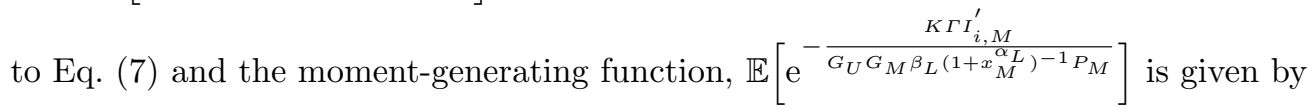

$$
\begin{aligned}
& \mathbb{E}\left[\mathrm{e}^{-\frac{\Gamma I_{i, M}^{\prime}}{G_{U} G_{M} \beta_{L}\left(1+x_{M}^{\alpha} L\right)^{-1}}}\right]=\mathbb{E}\left[\mathrm{e}^{-\frac{\Gamma \sum_{l \neq m}\left|h_{l ; i}\right|^{2} T_{l ; i} L\left(x_{M}\right)}{G_{U} G_{M} \beta_{L}\left(1+x_{M}^{\alpha} L\right)-1}}\right]=\prod_{l \neq m} \mathbb{E}\left[\mathrm{e}^{-\frac{\Gamma\left|h_{l ; i}\right|^{2} T_{l ; i} L\left(x_{M}\right)}{G_{U} G_{M} \beta_{L}\left(1+x_{M}^{\alpha}\right)-1}}\right] \\
& =\prod_{l \neq m}\left\{\mathbb{E}\left[\mathrm{e}^{-\frac{\Gamma\left|h_{l ; i}\right|^{2} T_{l ; i}\left(1+x_{M}^{\alpha}\right)^{-1}}{G_{U} G_{M}\left(1+x_{M}^{\alpha}\right)^{-1}}}\right] p_{L}\left(x_{M}\right)\right. \\
& \left.+\mathbb{E}\left[\mathrm{e}^{-\frac{\Gamma\left|h_{l ; i}\right|^{2} T_{l ; i} \beta_{N}\left(1+x_{N}^{\alpha}\right)^{-1}}{G_{U} G_{M} \beta_{L}\left(1+x_{M}^{\alpha}\right)^{-1}}}\right]\left(1-p_{L}\left(x_{M}\right)\right)\right\} \\
& =\left(\mathbb{E}\left[\frac{1}{1+\frac{\Gamma T_{i}\left(1+x_{M}^{\alpha_{L}}\right)^{-1}}{G_{U} G_{M}\left(1+x_{M}^{\alpha_{L}}\right)^{-1}}}\right] p_{L}\left(x_{M}\right)\right.
\end{aligned}
$$




$$
\left.+\mathbb{E}\left[\frac{1}{1+\frac{\Gamma T_{i} \beta_{N}\left(1+x_{M}^{\alpha_{N}}\right)^{-1}}{G_{U} G_{M} \beta_{L}\left(1+x_{M}^{\alpha_{L}}\right)^{-1}}}\right]\left(1-p_{L}\left(x_{M}\right)\right)\right)^{K-1}
$$

Then, Eq. (34) follows Eq. (40) based on the values of $T$ in Tab. 2.

It should be noted that, in order to avoid redundancy in this paper, we have omitted the details of the proof for $F_{N}\left(\Gamma, x_{M}\right)$, which can be obtained in a manner similar to that shown above.

\section{Achievable rate of interference lim- ited model}

Owing to the high density of SBSs, the system performance would be limited by the interferences caused by other SBSs and the MBS. Thus, for simplicity, we set $\sigma^{2}=0$, and the SINR thus becomes the SIR (Signal-to-Interference Ratio) in this section.

Definition 2 Given an SIR threshold $\Gamma$, a user is to be served by an SBS if $S I R_{S}=$ $\max _{1 \leqslant i \leqslant N_{U}} S I R_{i, S} \geqslant \Gamma$. Otherwise, the user would connect to the MBS.

Based on the user association scheme described in Definition 2, the average achievable rate with bandwidth $B$ is given by

$$
\begin{aligned}
& \mathbb{E}[B \operatorname{lb}(1+S I R)] \\
= & \mathbb{E}\left[B \operatorname{lb}\left(1+S I R_{S}\right)\right] P_{\mathrm{cov}, S}(\Gamma) \\
& +\mathbb{E}\left[B \operatorname{lb}\left(1+S I R_{M}\right)\right]\left(1-P_{\mathrm{cov}, S}(\Gamma)\right),
\end{aligned}
$$

where $S I R_{M}$ denotes the SIR with the MBS and user.

As discussed in Refs. [14,19], on considering an upper bound on the SIR, $\Gamma_{\max }$, Eq. (41) can be obtained as

$$
\begin{aligned}
& \mathbb{E}[B \operatorname{lb}(1+S I R)] \\
= & \frac{B}{\ln 2}\left(\int_{\Gamma}^{\Gamma_{\max }} \frac{P_{\mathrm{cov}, S}(t)}{1+t} \mathrm{~d} t P_{\operatorname{cov}, S}(\Gamma)\right. \\
& \left.+\int_{0}^{\Gamma} \frac{P_{\operatorname{cov}, M}(t)}{1+t} \mathrm{~d} t\left(1-P_{\operatorname{cov}, S}(\Gamma)\right)\right) .
\end{aligned}
$$

\section{Simulation results and analysis}

In this section, we present the numerical results obtained and validate the analytical expressions discussed in the previous sections.

For the simulations, we assume $G_{M}=30 \mathrm{~dB}$, $G_{S}=20 \mathrm{~dB}$, and $G_{U}=10 \mathrm{~dB}$. The side lobe gains are set as $g_{M}=g_{S}=g_{U}=-10 \mathrm{~dB}$. The half-power beamwidths of the main lobes are assumed to be $\theta_{M}=6^{\circ}, \theta_{S}=30^{\circ}$, and $\theta_{U}=10^{\circ}$. As in Ref. [20], we assume $\beta_{L}=\beta_{N}=1, \alpha_{L}=2$, and $\alpha_{N}=4$. Let $P_{M}=50 \mathrm{dBm}, P_{S}=30 \mathrm{dBm}$, and $\rho=20 \mathrm{~dB}$. Throughout this section, we also assume $N_{U}=10$ and $K=20$. In addition, we set the radius of the macro cell as $R=1000 \mathrm{~m}$.

The coverage probabilities of the SBSs are shown in Fig. 2. It should be noted that $R_{S}=\sqrt{1 / \pi \eta}$ represents the average radius of the small cells. As expected, the analytical results closely match those of the numerical simulations, which confirms the accuracy of the analytical expression for the coverage probability of the SBSs that was discussed in section 3. Moreover, it is interesting to observe that, on applying user beam selection, the coverage probability increases with the increase in the number of SBSs, which is different from the result obtained in Ref. [15].

In order to validate Property 1 in section 3, we present the analytical and numerical results for $N_{U}=5$ and 10 in Fig. 3. For the sake of comparison, the performance observed without applying beam selection (i.e., the scheme in Ref. [15]) is also shown in Fig. 3. It can be clearly observed that an improved performance can be obtained when we exploit the spatial diversity at the users, and the coverage probability increases with $N_{U}$.

In Fig. 4, we present the analytical average achievable rates when $R_{S}=30 \mathrm{~m}$ and $80 \mathrm{~m}$ respectively. It should be noted that the results for $R_{S}=30 \mathrm{~m}$ 
are relatively lower than those for $R_{S}=80 \mathrm{~m}$, and the degradation is not noticeable when the value of $\Gamma$ is low. Thus, threshold setting might be crucial for optimizing the performance of the HetNet, which will be discussed in our future work.

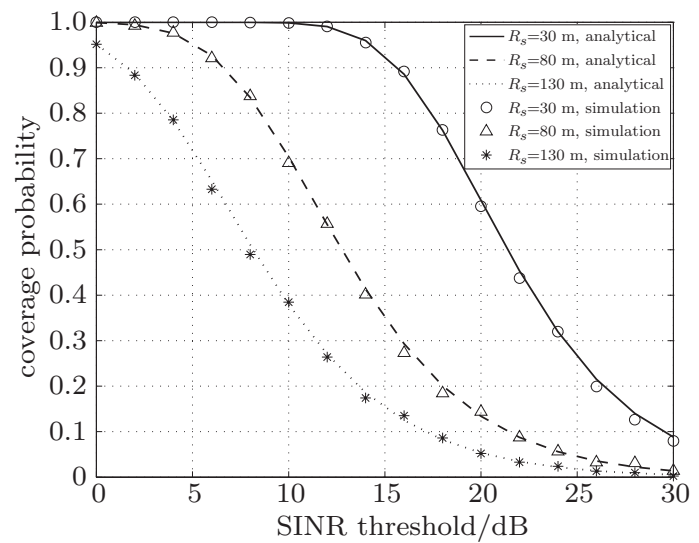

Figure 2 Coverage probabilities of SBSs in an mm-wave HetNet

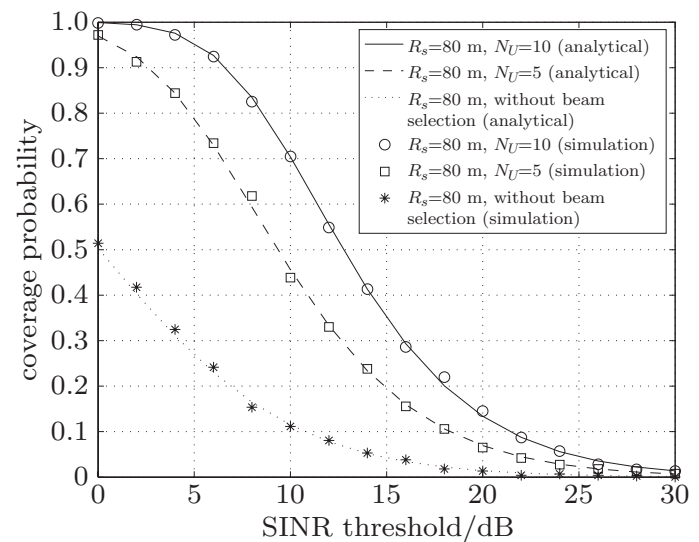

Figure 3 Coverage probabilities of SBSs with various values of $N_{U}$

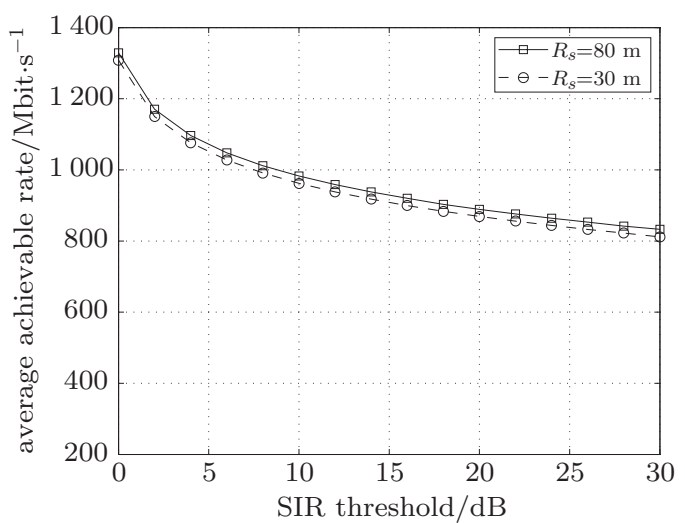

Figure 4 Achievable rate for a user with $B=100 \mathrm{MHz}$ when $R_{S}=30 \mathrm{~m}$ and $80 \mathrm{~m}$

\section{Conclusion}

In this paper, we analyzed the performance of an mm-wave HetNet. Expressions for the coverage probabilities of the SBSs and MBS with user beam selection were derived. Furthermore, on simplifying the SINR model, we analyzed the average achievable rate for a typical user in the HetNet. We used simulations to validate the results and illustrated that the coverage probability of the SBSs, with beam selection, increases as the number of deployed SBSs and user beams increases.

\section{References}

[1] W. Roh, J. Y. Seol, J. Park, et al. Millimeter-wave beamforming as an enabling technology for $5 \mathrm{G}$ cellular communications: theoretical feasibility and prototype results [J]. IEEE commun. mag., 2014, 52(2): 106-113.

[2] S. Sun, T. S. Rappaport, R. W. Heath, et al. MIMO for millimeter-wave wireless communications: beamforming, spatial multiplexing, or both? [J]. IEEE commun. mag., 2014, 52(12): 110-121.

[3] T. S. Rappaport, S. Sun, R. Mayzus, et al. Millimeter wave mobile communications for $5 \mathrm{G}$ cellular: It will work! [J]. IEEE access, 2013, 1: 335-349.

[4] Y. Roy, J. Y. Chouinard, S. A. Mahmoud. Selection diversity combining with multiple antennas for mm-wave indoor wireless channels [J]. IEEE j. sel. areas commun., 1996, 14(4): 674-682.

[5] M. Samimi, K. Wang, Y. Azar, et al. $28 \mathrm{GHz}$ angle of arrival and angle of departure analysis for outdoor cellular communications using steerable beam antennas in New York City [C]//IEEE 77th Vehicular Technology Conference (VTC Spring), 2013: 1-6.

[6] M. Marcus, B. Pattan. Millimeter wave propagation: spectrum management implications $[\mathrm{J}]$. IEEE microw. mag., 2005, 6(2): 54-62.

[7] Z. Y. Xiao, L. Bai, J. H. Choi. Iterative joint beamforming training with constant-amplitude phased arrays in millimeter-wave communications [J]. IEEE commun. lett., 2014, 18(5): 829-832.

[8] W. Choi, A. Forenza, J. G. Andrews, et al. Opportunistic space-division multiple access with beam selection [J]. IEEE trans. commun., 2007, 55(12): 2371-2380.

[9] J. H. Choi. Beam selection in mm-wave multiuser MIMO systems using compressive sensing [J]. IEEE trans. commun., 2015, 63(8): 2936-2947.

[10] Z. Y. Pi, F. Khan. An introduction to millimeter-wave mobile broadband systems [J]. IEEE commun. mag., 2011, 49(6): 101-107. 
[11] J. G. Andrews. Seven ways that HetNets are a cellular paradigm shift $[\mathrm{J}]$. IEEE commun. mag., 2013, 51(3): 136-144.

[12] J. G. Andrews, F. Baccelli, R. K. Ganti. A tractable approach to coverage and rate in cellular networks $[\mathrm{J}]$. IEEE trans. commun., 2011, 59(11): 3122-3134.

[13] R. W. Heath, M. Kountouris, T. Bai. Modeling heterogeneous network interference using Poisson point processes [J]. IEEE trans. signal process., 2013, 61(16): 4114-4126.

[14] T. Y. Bai, R. Vaze, R. W. Heath. Analysis of blockage effects on urban cellular networks [J]. IEEE trans. wireless commun., 2014, 13(9): 5070-5083.

[15] T. Y. Bai, R. W. Heath. Coverage and rate analysis for millimeter-wave cellular networks [J]. IEEE trans. wireless commun., 2015, 14(2): 1100-1114.

[16] L. Bai, T. Li, Z. Y. Xiao, et al. Performance analysis for SDMA mmWave systems: using an approximate closed-form solution of downlink sum-rate [J]. IEEE access, 2017, 5: 15641-15649.

[17] G. Z. Yao, N. Liu, Z. W. Pan, et al. Coverage and rate analysis for non-uniform millimeter-wave heterogeneous cellular network $[\mathrm{C}] / /$ th International Conference on Wireless Communications \& Signal Processing (WCSP), Yangzhou, China, 2016: 1-6.

[18] Z. G. Ding, P. Z. Fan, H. V. Poor. Random beamforming in millimeter-wave NOMA networks [J]. IEEE access, 2017, 5: 7667-7681.

[19] T. Brown. Cellular performance bounds via shotgun cellular systems [J]. IEEE j. sel. areas commun., 2000, 18(11): 2443-2455.

[20] T. S. Rappaport, F. Gutierrez, E. Ben-Dor, et al. Broadband millimeter-wave propagation measurements and models using adaptive-beam antennas for outdoor urban cellular communications [J]. IEEE trans. antennas propag., 2013, 61(4): 1850-1859.

\section{About the authors}

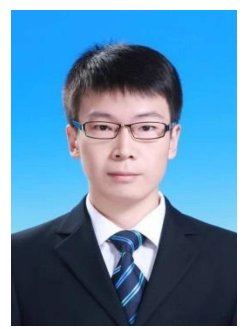

Tian Li received his B.E. degree from Hebei University, Baoding, China, in 2010, and his M.E. degree in Control Science and Engineering from Beijing Institute of Technology, Beijing, China, in 2013. He is now a Ph.D. candidate in information and communication engineering in School of Electronic and Information Engineering, Beihang University, Beijing, China. His current research interests cover areas of wireless communications, including multiple-input-multiple-output (MIMO) detections and millimeter-wave systems. (Email: tian.li@buaa.edu.cn)

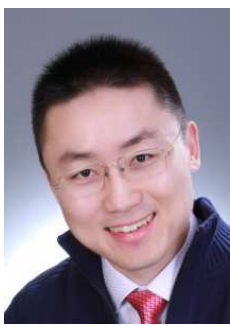

Lin Bai [corresponding author] received his B.Sc. degree in electronics and information engineering from Huazhong University of Science and Technology, Wuhan, China, in 2004, the M.Sc. (with distinction) degree in communication systems from the University of Wales, Swansea, U.K., in 2007, and the Ph.D. degree in advanced telecommunications from the School of Engineering, Swansea University, U.K., in 2010. He has been working with the School of Electronics and Information Engineering, Beihang University (Beijing University of Aeronautics and Astronautics, BUAA), Beijing, China, as an associate professor since 2011. Dr. Bai is the author of the books Low Complexity MIMO Detection and Low Complexity MIMO Receivers published by Springer in 2012 and 2014, respectively.

His research interests include signal processing of wireless communications, particularly multiple-input multiple-output systems, array/smart antenna, and lattice-based approaches. Dr. Bai received an IEEE communications letters exemplary reviewers certificate for 2012, the Best Paper Award from ICNS 2013 (Conference), and is a senior member of IEEE. Currently, he serves as an associate editor of IEEE access, an editor of KSII transactions on Internet and information systems, and the managing editor of Journal of communications and information networks. He also severed as a guest editor of International journal of distributed sensor networks from 2012 to 2014. (Email: 1.bai@buaa.edu.cn)

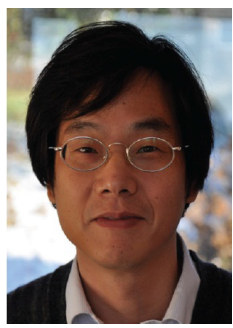

Jinho Choi was born in Seoul, Korea. He received his B.E. (magna cum laude) degree in electronics engineering in 1989 from Sogang University, Seoul, and M.S.E. and Ph.D. degrees in electrical engineering from Korea Advanced Institute of Science and Technology (KAIST), Daejeon, in 1991 and 1994, respectively. He is working with Gwangju Institute of Science and Technology (GIST) as a professor. Prior to joining GIST in 2013, he was with the College of Engineering, Swansea University, United Kingdom, as a professor/chair in wireless. His research interests include wireless communications and array/statistical signal processing. He authored two books published by Cambridge University Press in 2006 and 2010. Prof. Choi received the 1999 Best Paper Award for Signal Processing from EURASIP, 2009 Best Paper Award from WPMC (Conference), and is a senior member of IEEE. Currently, he is an editor of IEEE transactions on communications and had served as an associate editor or editor of other journals including IEEE communications letters, Journal of communications and networks, IEEE transactions on vehicular technology, and ETRI journal. (Email: jchoi0114@gist.ac.kr) 